\title{
Numerical study on thermocapillary convection in liquid bridge under surrounding shear gas stream
}

\author{
Ruquan Liang, Limin Kong, Fuqiang Yan , Di Bei and Shuo Zhang
}

Key Laboratory of National Education Ministry for Electromagnetic Processes of Materials, Northeastern University in Shen Yang, China.

\begin{abstract}
In this paper, a numerical simulation on the flow of liquid bridge and gas flow channel has been conducted to analyze the effect of airflow velocity on the isothermal liquid bridge free surface and the flow pattern in liquid bridge. The results show that the different pressure makes the lower part of liquid bridge shrink but makes the upper expand for the silicone oil liquid bridge of volume ratio $\mathrm{V}=1$, aspect ratio $\tau=1$. It will cause volatility in surface when shear gas stream just acting on the free surface of liquid bridge. When the shear gas stream acting on the free surface lasts $0.14 \mathrm{~s}$, the fluctuation of free surface reaches a steady state, and the fluctuation of surface is less than $0.5 \mu \mathrm{m}$. In the process of free surface wave, the maximum value of deformation usually appears in the bumps or the pits, and the deformation of the point near $\mathrm{z}=\mathrm{d} / 2$ is always zero.
\end{abstract}

\section{Introduction}

A new direction of studying floating zone method is using shear gas stream to restrain the effect of thermocapillary. The gas stream has launched application in the industry, such as the producing of high quality semiconductor ${ }^{[1]}$, microjets ${ }^{[2]}$, fiber and so on. On the other hand, the experimental studies on thermocapillary convection in liquid bridge were carried out mostly on the ground both at home and abroad, and some of them were space experiment. Although there were a lot of numerical calculations and theoretical analysis, it was difficult to find accurate stability analysis and numerical simulation $^{[3]}$. The reason was that the vast majority of studies used a simplified model and didn't take into account the deformation of liquid bridge free surface caused by shear gas stream, which set the free surface of liquid bridge to be non-deformable cylindrical or non-deformable surface by fluid volume and Young-Laplace equation ${ }^{[4-5]}$, affecting the accuracy of the findings. This article aims to study the deformation of liquid bridge surface caused by shear stream and the changes of temperature filed in internal flow field, which is under normal gravity conditions. Based on the basic knowledge of two-phase flow, we investigate the effect of air stream for isothermal liquid bridges. It directly services for the melt crystal growth of float zone on the ground, aiming at improving the quality of existing materials and processes, and it will further enriches the relevant research results of thermocapillary convection and small two-phase flow.

\section{The Establishment of Mathematical Models}

\subsection{The physical model}

The semi-floating zone model is shown in Fig. 1. The height and radius of the liquid bridge are $\mathrm{d}$ and $R_{0}$, respectively. Therefore, the aspect ratio of liquid bridge is $\tau=d / R_{0}=1$.

Geometrical scales used in the calculations are shown in Table 1. The upper and lower boundaries are the high and low temperature cylinders, respectively $\left(T_{h}\right.$ and $\left.T_{c}\right)$, $\Delta T=T_{h}-T_{c} \geq 0$. The $10 \mathrm{cSt}$ silicone is selected as the liquid, and the nitrogen is selected as the shear driven airflow. 


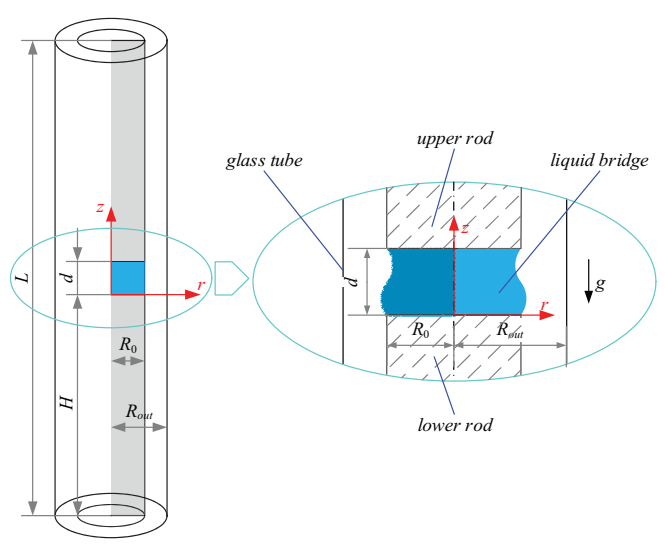

Fig 1. Semi-floating zone liquid bridge model

Table 1: Geometrical scales used in the calculations.

\begin{tabular}{|c|c|}
\hline Element & Size \\
\hline$R_{\text {out }}(\mathrm{mm})$ & 5 \\
\hline$R_{0}(\mathrm{~mm})$ & 3 \\
\hline$H(\mathrm{~mm})$ & 20 \\
\hline$d(\mathrm{~mm})$ & 3 \\
\hline$L(\mathrm{~mm})$ & 43 \\
\hline
\end{tabular}

\subsection{The governing equations}

The continuity equation:

$$
\nabla \cdot \vec{U}=0
$$

The momentum equation:

$$
\rho \frac{\partial \vec{U}}{\partial t}+\rho(\vec{U} \cdot \nabla) \vec{U}=-\nabla P+\rho v \nabla^{2} \vec{U}+\overrightarrow{F_{S}} \delta(\mathrm{r}-\mathrm{R})
$$

The energy equation:

$$
\frac{\partial T}{\partial t}+\vec{U} \cdot \nabla T-K \nabla^{2} T=0
$$

\subsection{Boundary conditions}

\subsubsection{The boundary conditions of wall}

The boundary conditions of velocity: The fluids satisfy the no-slip and do not penetrate boundary condition on the wall ${ }^{[6]}$, that is its velocity component meet:

$$
u_{r}=u_{z}=0
$$

The fluid velocity has two velocity components, that is the radial velocity and the axial velocity. In the above formula, $u_{r}$ represents the radial velocity, and $u_{z}$ represents the axial velocity.

The boundary conditions of temperature: In this study, the solid wall is considered to be isothermal, and the temperature of liquid near the wall equals to the temperature of wall.

\subsubsection{The boundary conditions of free surface}

The boundary condition of free surface kinematic and dynamic $^{[7]}$

$$
\begin{aligned}
& \left(p^{g}-p^{l}\right)+\frac{2 \mu^{l}}{1+r_{\text {surf }}^{\prime 2}}\left[\frac{\partial u_{r}^{l}}{\partial r}+r_{\text {surf }}^{\prime 2} \frac{\partial u_{z}^{l}}{\partial z}+-r_{\text {surf }}^{\prime}\left(\frac{\partial u_{z}^{l}}{\partial r}+\frac{\partial u_{r}^{l}}{\partial z}\right)\right] \\
& =\sigma\left[\frac{r_{\text {surf }}^{\prime \prime}}{\left(1+r_{\text {surf }}^{\prime 2}\right)^{3 / 2}}-\frac{1}{r_{\text {surf }}^{\prime}\left(1+r_{\text {surf }}^{\prime 2}\right)^{1 / 2}}\right]+ \\
& \frac{2 \mu^{g}}{1+r_{\text {surf }}^{\prime 2}}\left[\frac{\partial u_{r}^{g}}{\partial r}+r_{\text {surf }}^{\prime 2} \frac{\partial u_{z}^{g}}{\partial z}+-r_{\text {surf }}^{\prime}\left(\frac{\partial u_{z}^{g}}{\partial r}+\frac{\partial u_{r}^{g}}{\partial z}\right)\right]
\end{aligned}
$$

The boundary conditions of free surface temperature: Two fluids meet the conditions of continuous temperature and heat flux at the free surface ${ }^{[8]}$ :

$$
\begin{aligned}
T^{g} & =T^{l} \\
\frac{k^{g}}{\left(1+r_{\text {surf }}^{\prime 2}\right)^{1 / 2}}\left(\frac{\partial T^{g}}{\partial r}-r_{\text {surf }}^{\prime} \frac{\partial T^{g}}{\partial z}\right) & =\frac{k^{l}}{\left(1+r_{\text {surf }}^{\prime 2}\right)^{1 / 2}}\left(\frac{\partial T^{l}}{\partial r}-r_{\text {surf }}^{\prime} \frac{\partial T^{l}}{\partial z}\right)
\end{aligned}
$$

\subsubsection{The boundary conditions of inlet}

The gas velocity at entrance remains constant:

$$
\begin{array}{r}
u_{r}^{g}=0 \\
u_{z}^{g}=\text { const }
\end{array}
$$

The gas temperature at entrance equals to the ambient temperature:

$$
T^{g}=T_{0}
$$

\section{Numerical results}

\subsection{The influence of shear airflow on isothermal liquid bridge}

The influence of gas stream $\left(u^{g}=1 \mathrm{~m} / \mathrm{s}\right)$ on liquid bridge has a changing process. The liquid bridge shows a concave-convex shape under the influence of gravity. The gas stream entered from the bottom of liquid bridge in the 
opposite direction of gravity, weakening the effect of gravity on the surface shape of liquid bridge.

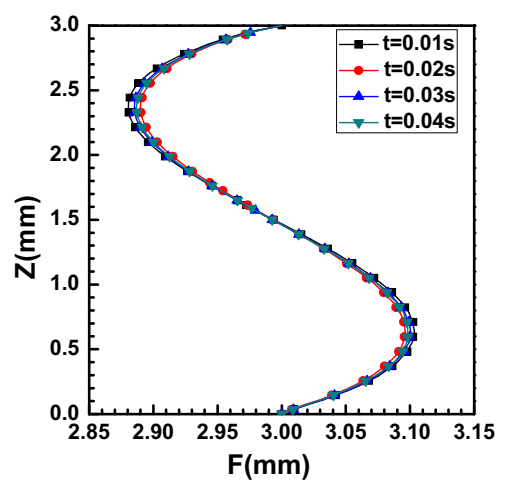

Fig 2. Initial interface shapes when the gas flow is directed against gravity $\left(P r=112, g=9.81 \mathrm{~m} / \mathrm{s}^{2}, \Delta T=0 \mathrm{~K}, u_{g}=1 \mathrm{~m} / \mathrm{s}, T_{a m b}=T_{0}\right.$, $\tau=1, V=1)$

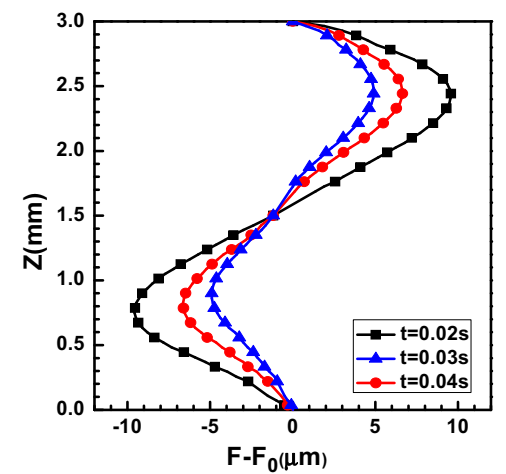

Fig 3. Initial dynamic interface deformation when the gas flow is directed against gravity $\left(P r=112, g=9.81 \mathrm{~m} / \mathrm{s}^{2}, \Delta T=0 \mathrm{~K}\right.$, $\left.u_{g}=1 \mathrm{~m} / \mathrm{s}, \mathrm{T}_{\mathrm{amb}}=\mathrm{T}_{0}, \tau=1, V=1\right)$

As shown in Fig 2, the free surface of liquid bridge changes significantly while the shear gas stream just acts on it. The convex surface reduces toward the inside, and the concave surface extends toward the outside, which is the effect of shear gas stream direct action.

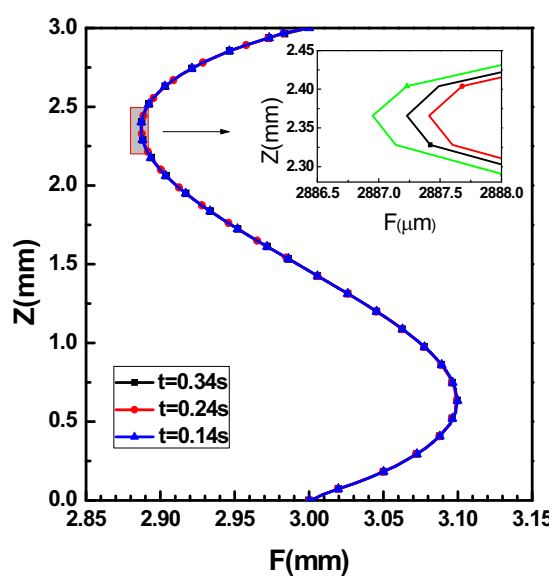

Fig 4. Interface shapes when the gas flow is directed against gravity for different times $\left(P r=112, g=9.81 \mathrm{~m} / \mathrm{s}^{2}, \Delta T=0 \mathrm{~K}\right.$, $\left.u_{g}=1 \mathrm{~m} / \mathrm{s}, T_{a m b}=T_{0}, \tau=1, V=1\right)$

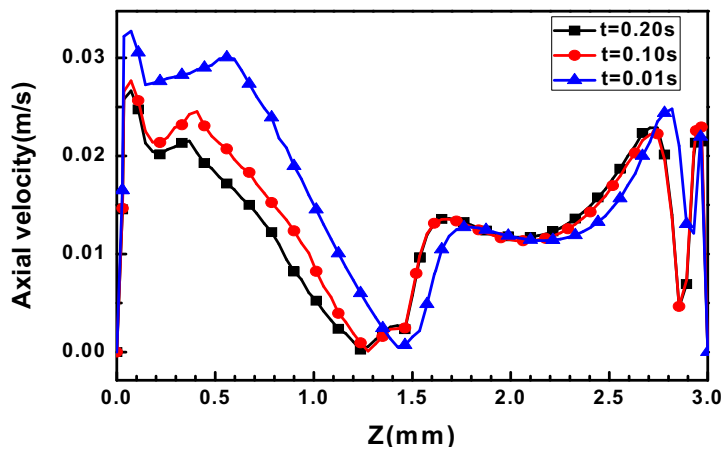

Fig 5. Interface velocity when the gas flow is directed against gravity for different times, $\left(P r=112, g=9.81 \mathrm{~m} / \mathrm{s}^{2}, \Delta T=0 \mathrm{~K}\right.$, $\left.u_{g}=1 \mathrm{~m} / \mathrm{s}, T_{a m b}=T_{0}, \tau=1, V=1\right)$

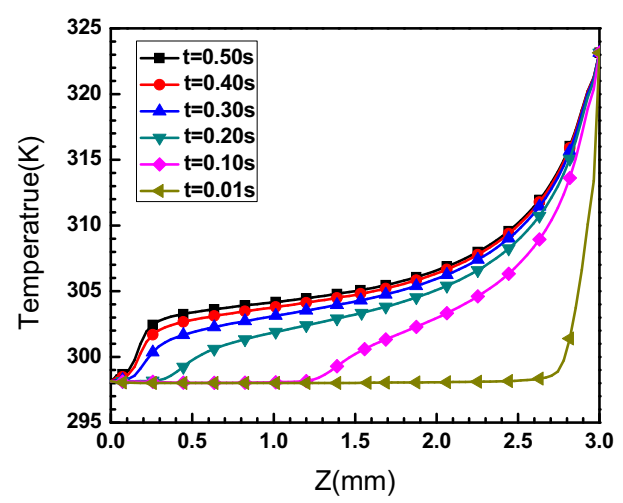

Fig 6. Interface temperatures when the gas flow is directed against gravity for different times $\left(P r=112, g=9.81 \mathrm{~m} / \mathrm{s}^{2}\right.$, $\left.\Delta T=25 \mathrm{~K}, u_{g}=1 \mathrm{~m} / \mathrm{s}, T_{a m b}=T_{0}, \tau=1, V=1\right)$

Fig 3 shows the size of the initial time of deformation, and we can get from the figure that the value of deformation reaches about $10 \mu \mathrm{m}$ both in the pits and the most bumps, while the deformation is essentially zero in the liquid bridge height of $\mathrm{z}=1.5$ around.

From Fig 4, we can know that the deformation of free surface reaches equilibrium after the shear gas stream acting for some time. The maximum deformation still occurs at the most bumps or best pits, but the free surface fluctuation is always smaller than $0.5 \mu \mathrm{m}$. Within this range, we can consider it reaches a steady state. To achieve this status requires more than $0.14 \mathrm{~s}$.

Fig 5 shows the distribution of the axial velocity of free surface. The axial velocity distribution of free surface has no obvious rules, and the maximum value occurs at a height of about $0.5 \mathrm{~mm}$, which should be the protruding part of liquid bridge facing the gas stream section. Under the direct action of liquid bridge, the gas stream nearly vertically acts on the surface. The minimum velocity occurs at the middle of liquid bridge. Because the liquid bridge is less directly affected and the counteract of deformation of the upper and lower side of liquid bridge, the velocity in here is the smallest.

Fig 6 shows the free surface temperature in different time. The flow of thermocapillary convection makes the heat transferred to the lower cylinder surface. The temperature in the vicinity of the upper and lower cylindrical surface changes more obvious. 


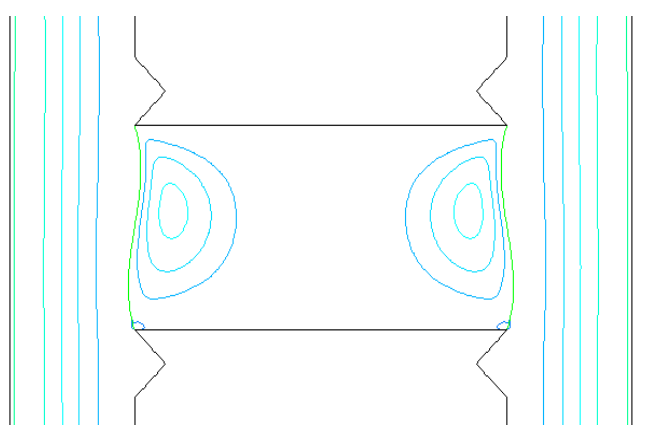

Fig 7. Streamline in two phase when the gas flow is directed against gravity $\left(P r=112, g=9.81 \mathrm{~m} / \mathrm{s}^{2}, \Delta T=25 \mathrm{~K}, u_{g}=1 \mathrm{~m} / \mathrm{s}\right.$, $\left.T_{a m b}=T_{0}, \tau=1, V=1\right)$

As shown in Fig 7, internal liquid bridge shows unit cell flow when the gas flow in the opposite direction of gravity. The unit cell near the free surface is like a shell. This is the collective effect of tangential air flow and thermocapillary convection.

\section{Conclusions}

In this paper, the silicone oil of $10 \mathrm{cSt}$ is used as the research object, and the ratio of height to diameter of $10 \mathrm{cSt}$ is 1 , and its volume ratio is 1 . The deformations of free surface caused by shear gas stream and its inhibiting effect to thermocapillary convection have been studied. The following conclusions have been achieved.

1. When shear gas stream acts on the free surface of liquid bridge, it will first cause the deformation of free surface. The deformation is comparatively large in the initial moments. After a period of time, the deformation of free surface reaches a steady state, and the deformation is less than $0.5 \mu \mathrm{m}$. It takes about $0.14 \mathrm{~s}-0.30 \mathrm{~s}$ to reach steady state and the time increases along with the increase of gas stream intensity.

2. For the isothermal liquid bridges, the deformation of liquid surface has certain rules. The deformation of free surface at the point near $\mathrm{z}=\mathrm{d} / 2$ is zero. The free surface has the maximum deformation at the most humps and the most concave. When the gas stream is in the opposite direction of gravity, the convex of free surface shrinks while the concave of free surface expands.

\section{Acknowledgment}

The present work is supported financially by the National Natural Science Foundation of China under the grants of 51376040 and 11072057.

\section{References}

1. Machida N, Hoshikawa K, Shimizu Y, Journal of Crystal Growth, The effects of argon gas flow rate and furnace pressure on oxygen concentration in Czochralski silicon single crystals grown in a transverse magnetic field. 4, $532-540(2000)$
2. Ganán-Calvo A M, Montanero J M, Phys Rev E Stat Nonlin Soft Matter Phys, Revision of capillary cone-jet physics: electrospray and flow focusing. 6, 1019-1027 (2009)

3. Hashimoto T, Kousaka Y, Ueno I, Jaxa Research \& Development Report, Numerical Simulation of Marangoni Convection in Consideration of Free Surface Displacement. 4, 2003-2004 (2005)

4. Wang A, Kamotani Y, Yoda S, International Journal of Heat and Mass Transfer, Oscillatory thermocapillary flow in liquid bridges of high Prandtl number fluid with free surface heat gain. 21, 4195-4205 (2007)

5. Sim B, Zebib A, Journal of Thermophysics and Heat Thansfer, Thermocapillary convection in liquid bridges with underformable curved surface. 4, 553-561 (2002)

6. Lappa M, John Wiley \& Sons, Thermal convection: patterns, evolution and stability. 15-16(2009)

7. Shevtsova V, Mialdun A, Kawamura $\mathrm{H}$ et al, Experimental benchmark, Fluid Dynamics \& Materials Processing, Onset of hydrothermal instability in liquid bridge. 1, 1-27 (2010)

8. Tiwari S, Nishino K, Fluid Dynamics \& Materials Processing, Effect of confined and heated ambient air on onset of instability in liquid bridges of high Pr fluids. 1, 109-136 (2010) 